\title{
Réplica al Dr. Palencia Herrejón
}

\section{Sr. Director:}

Hemos leído, con marcado interés, los comentarios de E. Palencia sobre nuestro original «Sesgo de retraso en el ingreso en la unidad de cuidados intensivos como causa de mal pronóstico o lead time bias» y le agradecemos, ante todo, el interés mostrado hacia nuestro trabajo.

Dicho esto, creemos que sus comentarios, aunque impecables desde un punto de vista metodológico (y para los que no tenemos más respuesta que estar de acuerdo) adolecen de una falta de coincidencia en la perspectiva de análisis.

Nuestra conclusión en dicho trabajo es que «la cuestión del sesgo de retraso en el ingreso en UCI sigue siendo una asignatura pendiente de adecuado estudio, y deben realizarse amplios estudios adicionales que permitan establecer la verdadera importancia y consecuencias de su prevención». Y en ello nos reafirmamos.

Nuestra intención no fue nunca demostrar que el retraso en el ingreso no tuviera importancia para el resultado intermedio (mortalidad) de la asistencia al paciente crítico. Nuestro objetivo fue constatar si, como había afirmado Dragsted ${ }^{2}$ (y no discutido por nadie posteriormente), el hecho del retraso se asociaba a una disminución de la fiabilidad de la estimación del riesgo de muerte de los pacientes en esa situación clínica.

A este respecto, y sólo a este respecto, es sorprendente que, sospechando que el factor sesgo de retraso (lead time bias) pudiera tener importancia en la estimación de mortalidad esperada, ninguno de los sistemas descritos, con posterioridad a APACHE II, incorporaron esa variable, y no ha sido hasta la publicación de SAPS 3 que ese retraso se tiene en consideración, y expresado en intervalos de días (> 14, 14-28, $>28$ ).

En nuestro conocimiento, sólo EPEC, descrito por Padrón et $\mathrm{al}^{3}$, incluye esta variable, y por ello despertó nuestro interés, aunque después comprobáramos la mala calibración del sistema y abandonáramos su uso $^{4}$.

¿Por qué? No es fácil dar una respuesta a esa pregunta, porque el retraso en el ingreso es un concepto variable que depende de la organización asistencial de cada centro. Así, en el nuestro, cuando se detecta a un paciente en situación crítica y no se lo puede ubicar en UCI (habitualmente por falta de cama disponible y en tanto no se libera ésta), la asistencia a dicho paciente es asumida por el equipo de intensi- 
vistas, que se desplaza y permanece junto al paciente hasta que su ingreso en UCI es posible. Esta es una de las fortalezas de nuestro sistema organizativo funcional y la causa probable de que la mortalidad de los pacientes con retraso en su ingreso en UCI no tenga un final tan infausto como en otras series. Incluso la cita de Chalfin et $\mathrm{al}^{5}$, aportada por nuestro corresponsal y aún no conocida cuando hicimos nuestro estudio y remitimos nuestro original, no especifica plenamente qué tipo de atención y prestada por quién reciben los pacientes que sufren retraso en su ingreso.

Otro motivo de nuestro interés fue suponer que hay diferencia entre lo que nosotros denominamos retraso fisiopatológico y retraso logístico, suponiendo que las diferencias de resultados respecto al supuesto se debían al primer grupo de pacientes, aunque no pudiéramos demostrarlo, probablemente por los motivos metodológicos argumentados por el Dr. Palencia.

Como resumen, y sin pretender enmendar los comentarios de nuestro respetado Eduardo, es evidente que el retraso en la asistencia efectiva de los pacientes críticos puede (¿debe?) asociarse a un peor resultado que el estimado, pero sigue estando pendiente el estudio que lo demuestre de forma fehaciente en nuestro ambiente (no en series procedentes de países con modelos asistenciales tan distintos del nuestro) y di- ferenciando adecuadamente la causa del retraso en el ingreso.

RICARDO ABIZANDA CAMPOS

Y BÁRBARA VIDAL TEGEDOR

Servicio de Medicina Intensiva. Hospital Universitario Asociado General de Castelló. Castelló de la Plana. España.

\section{BIBLIOGRAFÍA}

1. Vidal Tegedor B, Gómez Mico M, Abizanda Campos R, Álvaro Sánchez R, Berenguer Muncharaz A, Mateu Campo L, et al. Sesgo de retraso en el ingreso en la unidad de cuidados intensivos como causa de mal pronóstico o lead time bias. Med Intensiva. 2008;32:272-6.

2. Dragstedt L, Jorgensen J, Jensen NH, Bonsing E, Jacobsen E, Knaus WA, et al. Interhospital comparison of patient outcome from intensive care: importance of lead-time-bias. Crit Care Med. 1989;17:418-22.

3. Padrón Sánchez A, Puga Torres M, Peña Dorado R, Bravo Pérez R, Quiñones Zamora A. Escala pronóstica del enfermo crítico (EPEC). Propuesta de una nueva escala predictiva [citado Jun 2007]. http://bvs.sld.cu/revistas)mie/vol1_1_02/mie03102.htm

4. Abizanda R, Padrón A, Vidal B, Mas S, Berenguer A, Madero $\mathrm{J}$, et al. Estimación pronóstica en enfermos críticos (EPEC). Validación de un nuevo y simple sistema de estimación pronóstica de supervivencia en unidades de cuidados intensivos. Med Intensiva. 2006;30:101-8.

5. Chalfin DB, Trziak S, Likourezos A, Baumann BM, Dellinger RP. DELAY-ED study group. Impact of delayed transfer of critically ill patients from the emergency department to the intensiva care unit. Crit Care Med. 2007;35:1477-83. 